\title{
Musical and Photographic Stimulations on the Symptomatology of Alzheimer's Disease in Elderly
}

\author{
Jamylly Ferreira Targino Silva1 ( ${ }^{\circ}$, Mayara Leite Alves da Silva1 ( ${ }^{\circledR}$, \\ Maykon Wanderley Leite Alves-da-Silva1 ${ }^{(}$), Douglas Nijenhuis de Castro ${ }^{2}{ }^{(0)}$,

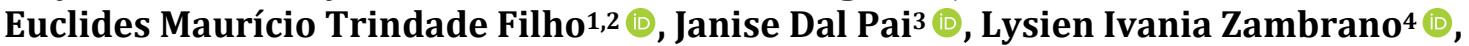 \\ Valtuir Barbosa Felix 2,5 ㅁ, Elionai Dias Soares², Camila Conceição Luz Soares², \\ Albérico José de Moura Saldanha Filho ${ }^{2,6}$ (), Magnúcia de Lima Leite ${ }^{1}$ (i), \\ Emanuel Guilherme de Almeida Carvalho ${ }^{7}$ (D), Matheus Santos Freitas ${ }^{1}$ (D), \\ José Claudio da Silva ${ }^{1,2 *}$
}

${ }^{1}$ State University of Health Sciences of Alagoas (UNCISAL), Maceió, Brazil

${ }^{2}$ CESMAC University Center, Maceió, Brazil

${ }^{3}$ Federal University of São Paulo (UNIFESP), São Paulo, Brazil

${ }^{4}$ Facultad de Ciencias Medicas, Universidad Nacional Autónoma de Honduras, Tegucigalpa, Honduras

${ }^{5}$ Division of Therapeutic Support and Diagnosis of HUPAA/UFAL/EBSERH, Maceió, Brazil

${ }^{6}$ Tiradentes University Center (UNIT-AL), Maceió, Brazil

${ }^{7}$ Faculdade Tiradentes (FITS), Recife, Brazil

Email: ${ }^{*}$ jcsneuroc1@gmail.com

How to cite this paper: Silva, J.F.T., da Silva, M.L.A., Alves-da-Silva, M.W.L., de Castro, D.N., Filho, E.M.T., Pai, J.D., Zambrano, L.I., Felix, V.B., Soares, E.D., Soares, C.C.L., de Moura Saldanha Filho, A.J., de Lima Leite, M., de Almeida Carvalho, E.G., Freitas, M.S. and da Silva, J.C. (2020) Musical and Photographic Stimulations on the Symptomatology of Alzheimer's Disease in Elderly. World Journal of Neuroscience, 10 , 155-165.

https://doi.org/10.4236/wjns.2020.104017

Received: August 18, 2020

Accepted: November 6, 2020

Published: November 9, 2020

\begin{abstract}
Background: Alzheimer's disease is a progressive and neurodegenerative disorder characterized by deterioration of brain functions. Psychosocial interventions such as music and photographic stimuli may contribute to cognitive and sensory exercise or rehabilitation. Objective: Evaluate musical and photographic stimuli in elderly people with Alzheimer's disease. Methods: It is an uncontrolled clinical trial, before-after type, eleven subjects composing the sample. Participants were submitted to Katz Index, Autobiographical Memory Test, GDS-15, Mini-Mental State Examination. The collection instruments were used on the first and last day of intervention, individually. There were 2 sessions in the week with duration of 50 minutes totaling 20 sessions. Data were analyzed by descriptive and inferential statistics and as variables expressed as mean standard deviation. Results: There was a significant effect of cognitive activity $(\mathrm{P}<0.01)$, it was observed that a small advance $(\mathrm{P}=0.05)$ of the daily life activities. With the analysis of GDS-15, it is verified that they are more effective for the increase of the previous self-estimation of the therapy $(\mathrm{P}=0.94)$. In the application of the AMT, it is observed that there was a bet-
\end{abstract}


Copyright (C) 2020 by author(s) and Scientific Research Publishing Inc. This work is licensed under the Creative Commons Attribution International License (CC BY 4.0).

http://creativecommons.org/licenses/by/4.0/

\section{Open Access}

ter recruitment of the memory for the neutral words. Conclusion: Musical and photographic stimuli had a positive effect on the cognitive level, daily life activities and autobiographical memory in seniors with Alzheimer's disease.

\section{Keywords}

Alzheimer's Disease, Health of the Elderly, Depression, Memory

\section{Introduction}

Alzheimer's disease $(\mathrm{AD})$ is a neurodegenerative and progressive disorder characterized by deterioration of brain functions such as attention, orientation, language, especially memory, which causes serious consequences for patients' quality of life [1]. This is due to the formation of neuritic plaques and tangles of neurofibrils, which damages cholinergic synapses and depletes the functionality of, for example, the limbic system, neurons of the cerebral cortex, tonsils and the base of the frontal cortex [2].

According to the International Association of Alzheimer's Disease, 35.6 million people have been living with AD worldwide since the period of 2010 and this estimate tends to grow exponentially every 20 years reaching 65.7 million in 2030 and 115.4 million by 2050 [3]. According to the World Health Organization, it is the most frequent dementia among the elderly with approximately $60 \%$ to $70 \%$ of the world's cases, reaching 1.2 million Brazilians [4].

This pathology represents a risk factor for depression due to neuropathological changes in the hippocampus, genetic causes, psychological reactions due to loss of cognitive functions as well as social isolation [5]. This associated with mild dementia, can cause in the patient behavioral change, such as aggression and suicide and making evident the need for complementary treatments [6].

Depression is common in people with $\mathrm{AD}$ and is associated with an increased risk of institutionalization and mortality. Understanding the risk factors for depression during Alzheimer's progression is critical to the correct management of your treatment [7]. Psychosocial interventions such as musical stimuli may contribute to maintaining or rehabilitating functional, emotional, and social cognitive and sensory abilities and thus reduce behavioral severity [8].

The use of music and photographs as non-pharmacological therapy is being widely used in these patients. Musical stimuli provide improvement of mood, sleep and depressive state, since music activates dopaminergic pathways, excitation and neural connection [9]. Moreover, personal memories could be preserved with the use of music in patients with $\mathrm{AD}$, and thus provide an interesting way to stimulate autobiographical memory [10].

In this perspective, a short protocol of musical stimuli can be an alternative to improve emotional variables and memory in patients with AD. The application of this therapy decreases the level of stress and significantly reduces depression 
[11]. When associated with photographic stimuli, it is of great value in the treatment of these patients, since the photographs have the singular fact of establishing the link between the past and the present, which necessitates the memory evocation [12].

This study was performed to observe the effects of photographic and musical stimuli on activities of daily life (ADL), depression and autobiographic memory in patients with $\mathrm{AD}$, and consequently, if there is improvement in quality of life contributing to reduce the evolution of cognitive impairment and memory.

\section{Methods}

It is an uncontrolled clinical trial of the before-after type. All procedures were initiated only after approval by the ethics and research committee at the State University of Health Sciences of Alagoas-UNCISAL (protocol 2.154.926). The caregivers of the elderly signed the Free and Informed Consent Form (ICF), since the elderly had cognitive impairment. The sample was selected for convenience, composed of eleven people, eight females and three males, from September 2017 to January 2018, in total twenty individual sessions, lasting 50 minutes each. The interventions were carried out using old photographs of the elderly provided by caregivers, as well as images of the places they used to frequent. The musical style proposed for each elderly person was selected according to the caregivers' information. Initially in each session, eight photographs were exhibited, which can be repeated in the following sessions, and immediately afterwards, five strings of string with musical style of each elderly person were placed.

Included in the study were people with $\mathrm{AD}$, who had no genetic etiology and aged between 45 and 90 years. Exclusion criteria were people with Alzheimer's disease with serious problems, bedridden, visually impaired conditions and/or hearing; individuals with $\mathrm{AD}$ associated neurological disorders; and that they did not have relatives who could report the kinds of unpleasant songs, avoiding some kind of bad memory. Through recent medical reports, elderly people with severe $\mathrm{AD}$ were excluded from the study.

As variables of the study were: sex, age, schooling, cognition, depression, activities of daily living and autobiographical memory. For collection, five instruments were used: Geriatric Depression Scale (GDS 15) that tracks depression in the elderly [13] [14]; Mini-Mental State Examination (MMSE) that evaluates cognition and location of dementia [15]; Test of the work of translation of frontal and temporoparietal functioning pattern; Autobiographical Memory Test (AMT) and KATZ Index that assess autobiographical memory [16] and functionality in daily life activities [17], respectively.

\section{Statistical Analysis}

Statistic GraphPad package (Prism ${ }^{\circledR}$ 5.0) was used for statistical evaluation. Kolmogorov-smirnov test was executed, and comparisons was analyzed using 
the Wilcoxon test and the t-paired test when the dates were non-parametric and parametric respectively. Data are presented as mean \pm standard deviation (SD) and the significance level was established at $5 \%$ with a significant difference $(\mathrm{P}<$ $0.05)$.

\section{Results}

Our results show that of the 11 patients, 3 of them (27.3\%) are males and 8 of them $(72.7 \%)$ are females, average age among patients is 79 years and about $45.5 \%$ were over 80 years. The level of education was incomplete primary education, predominating in $36.4 \%$ of the cases. The time of diagnosis of the disease referring to an average of time in approximately 4 years, fashion of 5 years (36.4\%) and the median of 4 years, ranging from 1 to 7 years of disease occurrence.

Self-identification of patients in front of the mirror, nine were recognized (79\%) while two (21\%) were not identified (Figure 1). Patients who did not recognize themselves were patient number 2 and 5 . These patients had been diagnosed with Alzheimer's for 5 years and 3 years, respectively. In this sense, it is believed that the non-recognition of their own image is not related to the severity of the disease, since patients with more years of diagnosis were recognized. However, patients who did not identify themselves had the same levels of schooling, complete elementary education, and thus, the relation of the area of the brain that processes and recognizes images in the teaching-learning process.

The results allowed to describe the effects of music and photographic stimuli for the elderly in four thematic evaluation axes, MMSE (Mini-Mental State Examination) that evaluates to cognitive function. Figure 2 shows the cognitive level of the patients evaluated by the MMSE at the beginning and end of the interventions. Our results show that there was a significant increase in cognitive activity.

In Figure 3 are the results distributed through the evaluation of the KATZ, which assesses the functional capacity of the elderly, index of the beginning and end of the 20 sessions of musical and photographic stimuli in the patients. It is observed that there was a small increase $(\mathrm{P}=0.05)$ in the $\mathrm{ADL}$, which characterizes

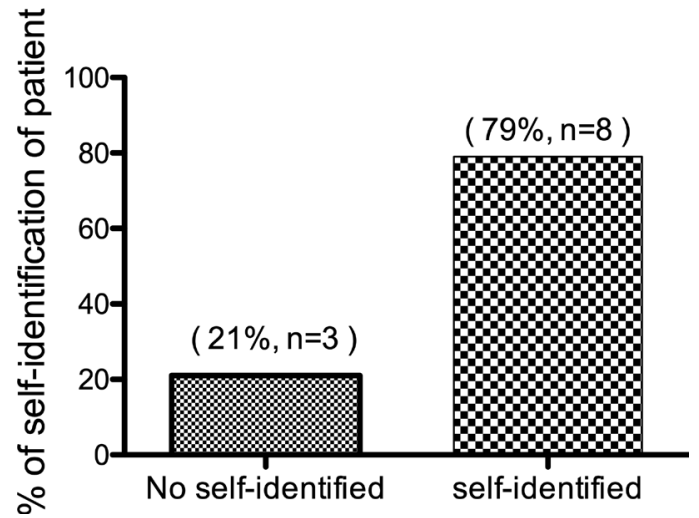

Figure 1. Evaluation of auto-recognition after test of measurement of patient self-identification in the mirror. 


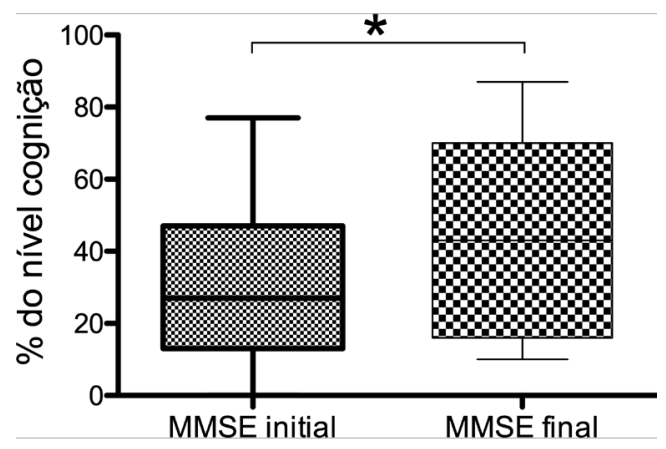

Figure 2. Cognitive level assessed by mini Mental State Examination (MMSE) after musical and autobiographical stimuli in patients with Alzheimer's disease. Unpaired T test. $\mathrm{P}$ $<0.01 . \mathrm{t}=3$, e df $=10 .(\mathrm{n}=11)$.

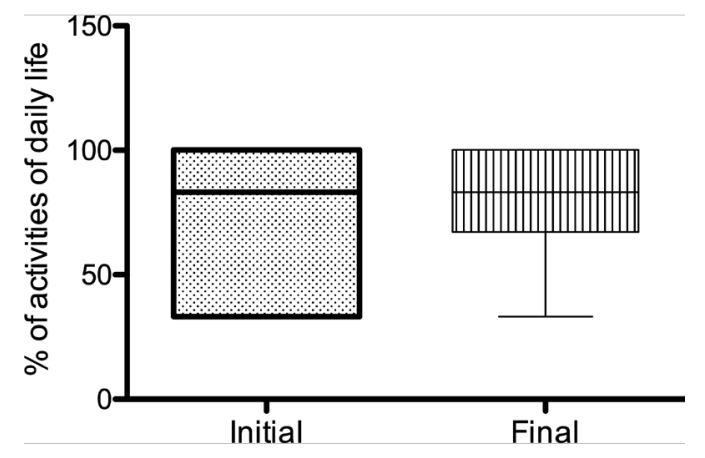

Figure 3. Measurement of daily life activity performed before and after musical and photographic stimuli in patients with Alzheimer's disease. $(n=11) . m \pm S D$.

a positive effect of the musical stimuli.

According to our results the measurement of the frontal and temporoparietal cortex of the cerebral functioning pattern performed before and after musical and photographic stimuli in patients with Alzheimer's disease showed that there was no significant difference $(\mathrm{P}=1.000)$ after the interventions. Do not were observed changes in the frontal and temporoparietal functioning of the cerebral cortex regions.

The GDS determines that when the total of points is greater than five, the patient is suspected of depression. It was evaluated before and after the interventions. In this context, it is observed in Figure 4, that the results demonstrate that a positive effect for the improvement of mood after the application of the technique $(\mathrm{P}=0.94)$.

Figure 5 shows the results of the AMT in the initial and final evaluation. It is observed that there was an increase of the memory for the neutral words, this shows a positive effect in the autobiographical memories after the interventions. Thus, the use of music and photographs as stimuli, rescue autobiographical memories in elderly people with $\mathrm{AD}$.

\section{Discussion}

From the data found, it was possible to observe that the age ranged from 68 to 89 


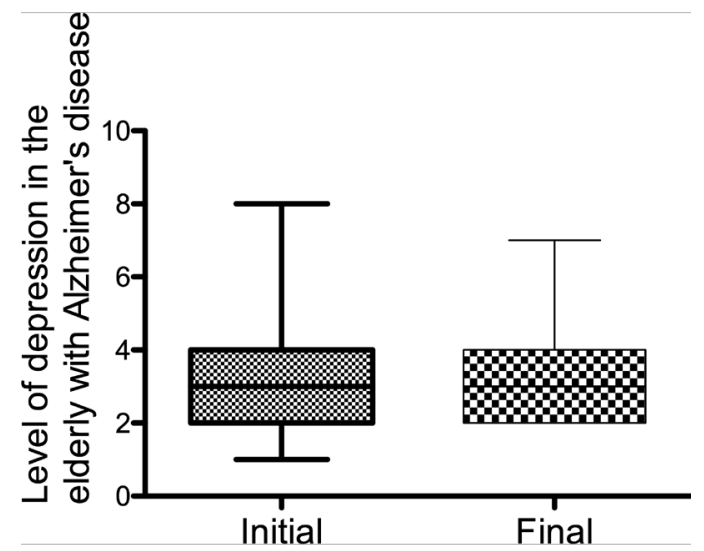

Figure 4. Effect of musical stimuli under the level of depression in patients with Alzheimer's disease. $(\mathrm{n}=11) . \mathrm{m} \pm \mathrm{SD}$.

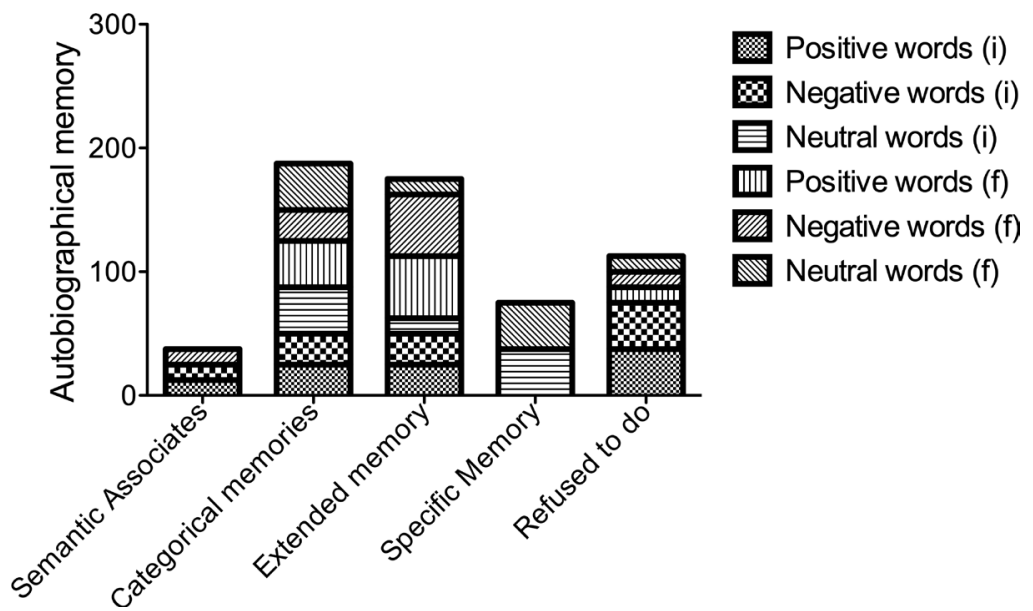

Figure 5. Effect of AMT before and after interventions under the level of cognition in patients with Alzheimer's disease.

years, which is similar to the Malta study [18], as well as an average age of 79 years [19]. However, it is in contrast to another study [20], which considers the prevalence of $\mathrm{AD}$ in individuals over 85 years of age. Regarding gender, what prevailed was the female, according to two studies [20] [21], which also affirm that this portion of the population is more likely to develop Alzheimer's.

It is also emphasized that there was a predominance of incomplete elementary school education, and only one subject in the research has complete higher education, compatible with the theory of cognitive reserve, in which people with complete higher education have a delay in the onset of Alzheimer's [22].

It is noted that receptive music therapy causes cognitive stimulation, allowing patients to recall memories and images [23]. The results of this study show that, despite cognitive loss, people with mild and moderate $\mathrm{AD}$ preserve the musical involvement and musical pursuit [24]. The regions normally involved in musical memory coding are well preserved in Alzheimer's disease [25]. That is, long-lasting musical memories seem to be largely preserved [24]. One study found that music with an ability to stimulate long-term and short-term memory in $\mathrm{AD}$ patients 
[26]. It is worth emphasizing, therefore, that the effects promoted by the musical and photographic stimuli obtained, mainly, relevance in the cognitive function of the participants of this study.

The Mini-Mental State Examination (MMSE) [15] is a tool for screening cognitive impairment most widely used in senescence [27]. However, it does not diagnose, being important to resort to other methods [28]. According to the results obtained in this study, only one participant regressed slightly, which corroborates with another study [29], when he states that music increases cognitive stimulation. A study carried out at a public university in Brazil, observed that all the elderly people considerably increased the score in the temporal orientation domain of the MMSE, after the introduction of stimulation activities at home [30].

Evidence of music-based interventions as a form of therapy results in benefits that potentiate cognitive, motor, emotional, and social relationships in older adults with $\mathrm{AD}$ [25]. Listening to regular and frequent music can facilitate cognitive recovery, emotional, neural, mood and quality of life recovery in people with moderate dementia [25].

The modified Geriatric Depression Scale (GDS-15) [13] [14], short version of the original, showed no improvement in mood and self-esteem, disagreeing with another study [25] when reporting that musical stimuli have an improvement effect mood, and excitement, temporarily causing a change in cognitive performance in attention or memory tasks in healthy older adults, as well as those with dementia.

A randomized study confirmed the effectiveness of music therapy in anxiety and depression in patients with $\mathrm{AD}$ and portrays that music therapy modifies the components of the disease through sensory, cognitive, affective and behavioral effects [31].

The subjects of this research were also submitted to Sidney Katz's Index of Independence of Daily Living Activities (KATZ), which evaluates the degree of functionality in Activities of Daily Life (ADL) [17] [32]. The autonomy of the elderly was evaluated considering their functional condition in performing six distinct basic activities of daily living (BADL) that were classified as independent and dependent. In this study, two participants improved scores on this scale at the end of interventions with music therapy and photographic stimuli, and the others remained the same, according to another study [33].

The loss of functional independence in self-care activities of patients with $\mathrm{AD}$ is greater than the physical and cognitive decline in the three stages of the disease [34]. Patients with the longest time of diagnosis did not, for the most part, obtain the worst functional performances in this test, disagreeing with two studies [35] [36], which state that, in general, functional performance is significantly associated with the severity of dementia.

In the Autobiographical Memory Test (AMT) what prevailed were the neutral words, with highlights for categorical and specific memories. In contrast, one 
study found low levels of specificity in neutral word scores [37]. In addition, familiar music evokes personal autobiographical memories in healthy and elderly youth, including mild to moderate $\mathrm{AD}[23]$.

With all this, music therapy can have a beneficial effect in relieving or reducing the main symptoms associated with dementia in terms of agitation, anxiety and depression [38]. In this way, music therapy improved some cognitive, psychological and behavioral changes in patients with Alzheimer's disease [39]. It is clear that the knowledge of a popular music seems to be relatively well preserved in patients with $\mathrm{AD}$ in the moderate stages and seems to be associated with the relatively preserved capacity of access to the autobiographical memories [40].

\section{Conclusion}

Alzheimer's disease causes considerable loss of memory that compromises functional independence, life history, and personal identity. This study shows that therapies with musical and photographic stimuli have positive effects on the symptoms of the pathology. More research is needed to investigate the long-term influence and corroborate the theoretical basis for the use of music and photography as a way to evoke memory in elderly people with chronic pathologies that affect the brain.

\section{Study Limitations}

The sample size was a limiting factor for being small. A larger sample would probably be able to better discriminate the results. In addition, there was no search for other factors that could interfere with the results, such as the onset of mood changes and the onset of signs and symptoms of Alzheimer's disease.

\section{Acknowledgements}

Cordially, I would like to thank the collaborators of the the Brazilian Alzheimer's Association of Maceió (ABRAZ), House for Old Age Luiza de Marillac and Center Specialized in Rehabilitation (CER III) from State University of Health Sciences of Alagoas-UNCISAL, Maceió, Brazil, who provided space and materials in their laboratories, contributing in an essential way to the accomplishment of the present work.

\section{Ethical Approval}

Ethical approval was given by the National Research Ethics Committee of Brazil (protocol number 2.154.926).

\section{Funding}

National Council for Scientific and Technological Development (CNPq). J. F. T. Silva is fellow from CNPq (Brazil)-[protocol number 129238/2017-1]. CNPq financed the research, the resource offered paid for the materials and expenses necessary to conduct the research. 


\section{Conflicts of Interest}

The authors declare no conflicts of interest regarding the publication of this paper.

\section{References}

[1] Brazilian Alzheimer's Association. About Alzheimer. http://www.abraz.org.br/index.php?page=alzheimer

[2] Nelson, P.T., Alafuzoff, I., Bigio, E.H., Bouras, C., Braak, H., Cairns, N.J., et al. (2012) Correlation of Alzheimer Disease Neuropathologic Changes with Cognitive Status: A Review of the Literature. Journal of Neuropathology \& Experimental Neurology, 71, 362-381. https://doi.org/10.1097/NEN.0b013e31825018f7

[3] Prince, M., Herrera, A.C., Knapp, M., Guerchet, M. and Karagiannidou, M. (2016) Alzheimer's Disease International: World Alzheimer Report. https://www.alz.co.uk/research/WorldAlzheimerReport2016.pdf

[4] World Health Organization. Dementia 2017. http://www.who.int/news-room/fact-sheets/detail/dementia

[5] Silva, R.H.C., Alencar, L.B.L., Alencar, E.T.L., Oliveira, G.F., Silva, R.A.C. and Alencar, A.B.L. (2012) Relationship between Depression and Dementia: A Focus on Alzheimer's Disease. Magazine of Psychology.

[6] Draper, B., Peisah, C., Snowdon, J. and Brodaty, H. (2010) Early Dementia Diagnosis and the Risk of Suicide and Euthanasia. Journal Alzheimer's \& Dementia, 6, 75-82. https://doi.org/10.1016/j.jalz.2009.04.1229

[7] Guetin, S., Charras, K., Berard, A., Arbus, C., Berthelon, P., Blanc, F., et al. (2013) An Overview of the Use of Music Therapy in the Context of Alzheimer's Disease: A Report of a French Expert Group. Dementia, 12, 619-634. https://doi.org/10.1177/1471301212438290

[8] Steck, N., Cooper, C. and Orgeta, V. (2018) Investigation of Possible Risk Factors for Depression in Alzheimer's Disease: A Systematic Review of the Evidence. Journal of Affective Disorders, 236, 149-156. https://doi.org/10.1016/j.jad.2018.04.034

[9] Peck, K.J., Girard, T.A., Russo, F.A. and Fiocco, A.J. (2016) Music and Memory in Alzheimer's Disease and the Potential Underlying Mechanisms. Journal of Alzheimer's Disease, 51, 949-959. https://doi.org/10.3233/JAD-150998

[10] Chevreau, P., Nizard, I. and Allain, P. (2017) Retrieval of Memories with the Help of Music in Alzheimer's Disease. Geriatrie et Psychologie Neuropsychiatrie du Vieillissement, 15, 309-318. https://doi.org/10.1684/pnv.2017.0689

[11] Ortí, J.E.R., García-Pardo, M.P., Iranzo, C.C., Madrigal, J.J.C., Castillo, S.S., Rochina, M.J. and Gascó, V.J.P. (2018) Does Music Therapy Improve Anxiety and Depression in Alzheimer's Patients? Journal of Alternative and Complementary Medicine, 24, 33-36. https://doi.org/10.1089/acm.2016.0346

[12] Gil, C.A. and Late, L.S.P.C. (2011) The Letter, Photo and Souvenir Workshop as a Group Psychotherapeutic Intervention with the Elderly. Changes-Health Psychology, 19, 19-27.

[13] Sheikh, J.I. and Yesavage, J.A. (1986) Geriatric Depression Scale (GDS): Recent Evidence and Development of a Shorter Version. Clinical Gerontologist. The Journal of Aging and Mental Health, 5, 165-173. https://doi.org/10.1300/J018v05n01 09

[14] Paradela, E.M.P., Lourenço, R.A. and Veras, R.P. (2005) Validação da escala de depressão geriátrica em um ambulatório geral. Revista de Saúde Pública, 39, 918- 
923. https://doi.org/10.1590/S0034-89102005000600008

[15] Folstein, M.F., Folstein, S.E. and Mchugh, P.R. (1975) “Mini-Mental State”: A Practical Method for Grading the Cognitive State of Patients for the Clinician. Journal of Psychiatric Research, 12, 189-198. https://doi.org/10.1016/0022-3956(75)90026-6

[16] Pergher, G.K. (2005) Avaliando a especificidade da memória autobiográfica: O teste de memória autobiográfica. Dissertation, Pontifical Catholic University of Rio Grande do Sul, Porto Alegre.

[17] Katz, S., Ford, A.B., Moskowitz, R.W., Jackson, B.A. and Jaffe, M.W. (1963) Studies of Illness in the Aged. The Index of ADL: A Standardized Measure of Biological and Psychosocial Function. JAMA, 185, 914-919. https://doi.org/10.1001/jama.1963.03060120024016

[18] Malta, T.M.X. (2012) Depressive Symptoms and Autobiographical Memory in Elderly People with Alzheimer's Dementia in the Mild and Moderate Stages. Doctoral Thesis, University of Beira Interior, Covilhã.

[19] Talmelli, L.F.D.S., Vale, F.D.A.C.D., Gratão, A.C.M., Kusumota, L. and Rodrigues, R.A.P. (2013) Alzheimer's Disease: Functional Decline and Stage of Dementia. Acta Paulista de Enfermagem, 26, 219-225. https://doi.org/10.1590/S0103-21002013000300003

[20] Thies, W. and Bleiler, L. (2011) Alzheimer's Association Report: 2011 Alzheimer's Disease Facts and Figures. Alzheimer's \& Dementia, 7, 208-244.

https://doi.org/10.1016/j.jalz.2011.02.004

[21] Soares, N.M., Pereira, G.M., Figueiredo, R.I.D.N., Soares, N.M., Almeida, R.M.M.D. and Portela, A.D.S. (2017) Economic Impact and Prevalence of Alzheimer's Disease in a Brazilian Capital. Science \& Health, 10, 133-138. https://doi.org/10.15448/1983-652X.2017.3.25036

[22] Voos, M.C., Mansur, L.L., Caromano, F.A., Brucki, S.M.D. and Valle, L.E.R. (2014) The Influence of Schooling on the Performance and Learning of Motor Tasks: A Literature Review. Physiotherapy and Research, 21, 297-304.

[23] Guetin, S., Portet, F., Picot, M.C., Pommié, C., Messaoudi, M., Djabelkir, L., et al. (2009) Effect of Music Therapy on Anxiety and Depression in Patients with Alzheimer's Type Dementia: Randomised, Controlled Study. Dementia and Geriatric Cognitive Disorders, 28, 36-46. https://doi.org/10.1159/000229024

[24] Cuddy, L.L., Sikka, R. and Vanstone, A. (2015) Preservation of Musical Memory and Engagement in Healthy Aging and Alzheimer's Disease. Annals of the New York Academy of Sciences, 1337, 223-231. https://doi.org/10.1111/nyas.12617

[25] Särkämö, T. (2017) Cognitive, Emotional, and Neural Benefits of Musical Leisure Activities in Aging and Neurological Rehabilitation: A Critical Review. Annals of Physical and Rehabilitation Medicine, 61, 414-418.

https://doi.org/10.1016/j.rehab.2017.03.006

[26] Kerer, M., Marksteiner, J., Hinterhuber, H., Mazzola, G., Kemmler, G., Bliem, H.R., et al. (2013) Explicit (Semantic) Memory for Music in Patients with Mild Cognitive Impairment and Early-Stage Alzheimer's Disease. Experimental Aging Research, 39, 536-564. https://doi.org/10.1080/0361073X.2013.839298

[27] Anthony, J.C., LeResche, L., Niaz, U., Von Korff, M.R. and Folstein, M.F. (1982) Limits of the "Mini-Mental State" as a Screening Test for Dementia and Delirium among Hospital Patients. Psychological Medicine, 12, 397-408. https://doi.org/10.1017/S0033291700046730

[28] Nitrini, R., Caramelli, P., Bottino, C.M.D.C., Damasceno, B.P., Brucki, S.M.D. and Anghinah, R. (2005) Diagnosis of Alzheimer's Disease in Brazil: Cognitive and Func- 
tional Evaluation. Recommendations of the Scientific Department of Cognitive Neurology and Aging of the Brazilian Academy of Neurology. Neuro-Psychiatry Archives, 63, 720-727. https://doi.org/10.1590/S0004-282X2005000400034

[29] Light Ltd. (2015) Music Therapy in the Quality of Life of Institutionalized Elderly. Master's Dissertation, Pontifical Catholic University of Rio Grande do Sul, Porto Alegre.

[30] Cruz, T.J.P., Sá, S.P.C., Lindolpho, M.C. and Caldas, C.P. (2015) Cognitive Stimulation for Elderly People with Alzheimer's Disease Performed by the Caregiver. Revista Brasileira de Enfermagem, 68, 510-516.

https://doi.org/10.1590/0034-7167.2015680319i

[31] Guetin, S., Portet, F., Picot, M.C., Pommié, C., Messaoudi, M., Djabelkir, L., et al. (2009) Effect of Music Therapy on Anxiety and Depression in Patients with Alzheimer's Type Dementia: Randomised, Controlled Study. Dementia and Geriatric Cognitive Disorders, 28, 36-46. https://doi.org/10.1159/000229024

[32] Duarte, Y.A.O., Andrade, C.L. and Lebrão, M.L. (2007) The Katz Index in Assessing the Functionality of the Elderly. USP School of Nursing Magazine, 41, 317-325. https://doi.org/10.1590/S0080-62342007000200021

[33] Taylor, L.O. and Dellaroza, M.S.G. (2010) The Reality of Care for the Elderly with Alzheimer's Disease: An Analysis from the Elderly Treated in Public Services. Seminar. Biological and Health Sciences, 31, 71-82.

[34] Zildan, M., Arcoverde, C., Araújo, N.B., Vasques, P., Rios, A., Iaks, J. and Deslandes, A. (2012) Motor and Functional Changes at Different Stages of Alzheimer's Disease. Magazine of Clinical Psychiatry, 39, 161-165. https://doi.org/10.1590/S0101-60832012000500003

[35] Camacho, A.C.L.F. and Coelho, M.J. (2010) Identification of Clinical of Alzheimer's Disease to the Development in Nursing Care. Journal of Nursing UFPE Online, 4, 517-523. https://doi.org/10.5205/reuol.647-7353-1-LE.0402201010

[36] Monaci, L. and Morris, R.G. (2012) Neuropsychological Screening Performance and the Association with Activities of Daily Living and Instrumental Activities of Daily Living in Dementia: Baseline and 18- to 24-Month Follow-Up. International Journal of Geriatric Psychiatry, 27, 197-204. https://doi.org/10.1002/gps.2709

[37] Pergher, G. and Stein, L.M. (2008) Recovering Autobiographical Memories: Evaluation of the Brazilian Version of the Autobiographical Memory Test. Psycho, 39, 6.

[38] Lopes, C.D.J., André, R., Sousa, L.M.M., Oliveira, I. and Silveira, T. (2019) Benefits of Music Therapy in the Elderly with Dementia: An Integrative Literature Review. Journal of Nursing Research, 45-59.

[39] Gallego, M.G. and García, G.J. (2017) Music Therapy and Alzheimer's Disease. Cognitive, Psychological and Behavioral Effects. Neurology (English Edition), 32, 300-308. https://doi.org/10.1016/j.nrleng.2015.12.001

[40] Basaglia-Pappas, S., Laterza, M., Borg, C., Richard-Mornas, A., Favre, E. and Thomas-Antérion, C. (2013) Exploration of Verbal and Non-Verbal Semantic Knowledge and Autobiographical Memories Starting from Popular Songs in Alzheimer's Disease. International Psychogeriatrics, 25, 785-795.

https://doi.org/10.1017/S1041610212002359 